\title{
Assessment of Malnutrition and Enteral Feeding Practices in Critically Ill in Neurosurgery ICU in Rural Teaching Hospital
}

\author{
Aditya Rameshbabu Devalla ${ }^{1}$, Hemant Deshpande ${ }^{2}$, Sanjot Ninave ${ }^{3}$, Rakesh Bhaisare ${ }^{4}$ \\ 1Department of Anaesthesiology, Jawaharlal Nehru Medical College (JNMC), Datta Meghe Institute of \\ Medical Sciences (DMIMS), Sawangi, Wardha, Maharashtra, India. ${ }^{2}$ Department of Critical Care Medicine, \\ Jawaharlal Nehru Medical College (JNMC), Datta Meghe Institute of Medical Sciences(DMIMS), Sawangi, \\ Wardha, Maharashtra, India. ${ }^{3}$ Department of Anaesthesiology, Jawaharlal Nehru Medical College (JNMC), \\ Datta Meghe Institute of Medical Sciences(DMIMS), Sawangi, Wardha, Maharashtra, India. ${ }^{4}$ Department of \\ Critical Care Medicine, Jawaharlal Nehru Medical College (JNMC), Datta Meghe Institute of Medical \\ Sciences(DMIMS), Sawangi, Wardha, Maharashtra, India.
}

\section{ABSTRACT}

\section{BACKGROUND}

Even after the recent advances and new technologies, prevalence of malnutrition has remained unchanged over the last two decades in India. Both overfeeding and underfeeding affect the outcome of critically ill patients. Hence the assessment of nutritional status becomes the important aspect in critically ill patients and with prolonged ICU stay. The objective of the study was to assess the nutritional status of critically ill patients with prolonged ICU stay before intervening with enteral feeds and parenteral feeds.

\section{METHODS}

This prospective, observational study was conducted in the Neurosurgical Intensive Care Unit of a tertiary care rural hospital in central India over a period of 6 months after the institutional ethical committee approval. The study included adult patients admitted for more than 72 hours in the Intensive Care Unit who were initiated on Ryles tube feeding.

\section{RESULTS}

Among 60 patients, 27 patients come under class A of the Subjective Global Assessment (SGA) Scale, 24 patients come under the class B. 9 patients came under class C.

\section{CONCLUSIONS}

Incidence of malnutrition was found to be $60 \%$ as per the of the Subjective Global Assessment (SGA) scale. Of these, $40 \%$ of patients were categorized under class B (mildly malnourished), $15 \%$ of patients were categorized under class C (moderate malnutrition).

\section{KEY WORDS}

Malnutrition, Critically Ill, Anaesthesia, Intensive Care, ICU
Corresponding Author: Dr. Hemant Deshpande, Assistant Professor, Department of Critical Care Medicine, Datta Meghe Institute of Medical Sciences, (DMIMS), Sawangi, Wardha,

Maharashtra, India.

E-mail: hemsdesh1980@gmail.com

DOI: $10.14260 / \mathrm{jemds} / 2020 / 568$

How to Cite This Article:

Devalla AR, Deshpande $H$, Ninave $S$, et al. Assessment of malnutrition and enteral feeding practices in critically ill in neurosurgery ICU in rural teaching hospital. J Evolution Med Dent Sci 2020;9(36):2610-2613, DOI: $10.14260 /$ jemds/2020/568

Submission 27-02-2020,

Peer Review 27-07-2020,

Acceptance 05-08-2020,

Published 07-09-2020.

Copyright (C) 2020 JEMDS. This is an open access article distributed under Creative Commons Attribution License [Attribution 4.0 International (CC BY 4.0)] 


\section{BACKGROUND}

The prevalence of malnutrition in cancer ranges between about 20 and $70 \%$. Diverse consequences of cancer patients are correlated with malnutrition, such as reduced quality of life, adverse injuries induced by chemotherapy and / or radiotherapy, worse prognosis and shorter overall survival. Malnutrition among hospitalized patients is associated with poor health outcomes. However, studies in the intensive care unit (ICU) that link malnutrition with poor clinical outcomes often have conflicting findings, due in part to inappropriate malnutrition diagnosis. Nutritional status plays a key role in the mortality of onco - logical patients, with research suggesting that about 20 per cent of deaths are associated with malnutrition and its effects. Serious or serious illness refers to the spectrum of life-threatening health or surgical conditions that most commonly involve hospitalization in an Intensive Care Unit (ICU). Extreme or critical disease applies to both the continuum of health or clinical disorders that present life threatening risks, and most commonly involve hospital stay in an ICU. Hormonal shifts arise to maintain the homoeostasis of the organism. Among certain symptoms, insulin tolerance and protein catabolism, such a situation also allows the individual to be vulnerable to severe nutritional risks. There are also some questions with patients with serious illness about nutritional therapy, while newly updated recommendations recommend nutritional therapy for those patients with an ICU stay longer than $48 \mathrm{~h}$. Latest findings also found that not every critical conditions can prosper from intensive nutritional therapy in the early stages of serious illness, and the only exception would be patients of greater nutritional risk. The correct and early detection of risk in ICU patients is of paramount importance for this cause. Incorporating the study of diets with processes.

Child hospitalization routine is important, regardless of its severity and the associated risks of morbidity and mortality. It is attributable to the likelihood of recognizing the prevalence of metabolic deficiencies, such as malnutrition and obesity, this could be associated with infections and / or other inappropriate social circumstances. Providing dietary assistance for chronically ill patients can be deemed as essential as every other step in health care. Nutritional risk may be described as a greater likelihood of developing negative consequences linked to the nutritional condition. Is the need for dietary assistance to include calorific requirements, absorb electrolytes and sustain the homoeostatic atmosphere. ${ }^{1}$ Nutritional support is required to provide the calorific needs, replace the electrolyte and preserve the homoeostatic environment. The therapies undergoes by patients with serious illness often affect nutritional support. Hypotension contributes to fluid administration and use of the vasopressor, all of which may conspire through either oedema or decreased blood flow to minimize gastrointestinal function.

Usage of high - dose opioids through injection may cause or worsen current ileus, while use of wide - spectrum antibiotics may contribute to diarrhoea with resulting loss of volume while electrolyte. The complexities in providing support in chronically sick individuals include unstable or missing means in governance, fluctuating demands and possible iatrogenic risks of the assistance selected. Despite good evidence that indicates that initiation of enteral feeds is beneficial in chronically ill patients, over half of all ICU patients worldwide remain significantly underfed. Limited statistics from Indian hospitals suggest specific low rates of feed in the admitted patients owing to inadequate baseline nutritional status. $^{2}$ Hospital feeding is commonly deemed difficult to eat in children, and is thus recommended in people requiring artificial ventilation that are seriously ill. Current ESPEN recommendations notice that medicinal diet treatment is required for certain ICU patients; sometimes for over 48 hours, because any seriously sick person staying in the ICU for over $48 \mathrm{~h}$ will be deemed at nutritional risk. Current ESPEN recommendations notice that medicinal diet treatment is required for certain ICU patients; sometimes for over 48 hours, because any seriously sick person staying in the ICU for over $48 \mathrm{~h}$ will be deemed at nutritional risk. ${ }^{3-9}$

The Subjective Global Assessment (SGA) is a nutrition evaluation tool that reflects on some aspects from medical background, such as recent weight change and food consumption, digestive problems with food consequences, cognitive capacity and stress strength, and subjective examination findings in muscle mass reserves. It is advised that SGA be used to identify patients who might gain from nutritional therapies, and it is still called a fixed exchange rate method for conducting these tests in hospital environment, particularly after nearly three decades. It is proposed that SGA be used to identify patients who might gain from dietary therapies, but it is still called a fixed exchange rate method for conducting these tests throughout hospital setting after almost 3 decades. ${ }^{10}$

Although nutritional therapy knowledge in ICU has improved over the last decade, certain basic measures are not well defined as the best option for nutritional screening or nutritional evaluation for such patients. Early diagnosis of a greater dietary risk or severe malnutrition patients is critical, because these patients are really the only ones who will profit from efficient and extensive pharmacological intervention. ${ }^{11}$

Early detection of high - risk and severely malnourished patients is therefore important because they are more likely to benefit from an immediate and more efficient dietary intervention, whether for enteral or oral nutrition. ${ }^{5}$

Qualitative metabolic evaluation is a therapeutic judgment - based measurement device that was widely used for therapeutic testing purposes to evaluate adult nutritional status, called a morbidity and mortality indicator. Qualitative metabolic evaluation is a therapeutic judgment - based measurement device that was widely used for therapeutic testing purposes to evaluate adult nutritional status, called a morbidity and mortality indicator. ${ }^{6}$ While other newly released, well - done research fail to answer important questions about dietary habits such as the delivery route, timing of diet implementation and the precise amount of energy and protein to be supplied. The purpose was to explore the risk of malnutrition among patients admitted to neurosurgical ICU. The main aim was to examine the level of deprivation and the secondary objective was to determine potential causes stopping the patients from meeting feeding targets. ${ }^{12}$ 


\section{METHODS}

This prospective, observational study was conducted in the Neurosurgical Intensive Care Unit (ICU) of a tertiary care rural hospital in central India over a period of 6 months after the institutional ethical committee approval. The study included adult patients admitted for more than 72 hours in the Intensive Care Unit who were initiated on Ryles tube feeding. A total of 60 patients were included in this study. The following patients were excluded from our study - patients with non functional gastrointestinal tract (e.g. Previous resection and anastomosis with short bowel syndrome, intestinal trauma), severe shock states i.e.; those on more than two vasopressors and those on short fasting periods i.e. $<24$ hours. After enrolment to the study, baseline demographic data, height, weight and diagnosis at the time of admission were noted. Laboratory values of CBC, KFT, LFT and PT - INR were recorded. All the patients were evaluated using Subjective Global Assessment score at the ${ }^{6}$ time of admission. The Subjective Global Assessment (SGA) is a questionnaire type tool, 68 of which is filled in on the bedside by every qualified health care professional planning to diagnose nutritional status by 69. It had been confirmed by Detsky et al.13 And demonstrated 70 capabilities to subjectively classify the possibility of worse clinical development in surgical patients associated with an aggravated nutritional status of 60 . In 72 ways, by the examiner's empirical study, the questions relating to weight loss history, Changes in food intake, 60 improvements in gastrointestinal and functional ability, metabolic demand associated with the 74 primary illnesses and physical examinations, the patient's nutritional status is graded as 75: (A) well - nourished, (B) moderately (or suspected of being) malnourished, or (C) 76 extremely malnourished. Since SGA is relatively simple, non - invasive and of low cost, it 77 became broadly used, including in non surgical patient groups ${ }^{14}$ With each patient, we reported the date on which Ryles tube (RT) feeds were started, along with explanations with delayed fee deeding. The explanations for the same have been noted in cases where the daily feeding targets were not achieved. It also reported the date at which feeds were stopped. ${ }^{15}$

Patients were followed up until they were released from the Intensive Care Unit or oral feeds were provided whichever they were earlier. It also noted the potential outcomes of patients (discharged home or death).

\section{Statistical Analysis}

The statistical analyses have been carried out using the Social Sciences Statistical System (SPSS), version 16 (SPSS Inc., Chicago, IL). The data are expressed on the basis of normal / abnormal distribution or number / percent as mean \pm SD and median (interquartile range).

\begin{tabular}{|ccc|}
\hline \multicolumn{3}{|c|}{ RESULT S } \\
\hline \\
\hline Variable & Category & No. of Patients \\
\hline \multirow{2}{*}{ Age } & $20-30$ yrs. & 4 \\
& $30-40$ yrs. & 12 \\
Gender & $40-50$ yrs. & 42 \\
& Male & 36 \\
& Female & 24 \\
\hline \multicolumn{2}{|c|}{ Table 1. Demography of the Study Population } \\
\hline
\end{tabular}

Table 1 shows Demography details of study Population total of 60 patients were included in this study all from the adult patients admitted for more than 72 hours in the Intensive Care Unit who were initiated on Ryles tube feeding. Out of 6036 parties were male while 24 were female; most of the study participants were from 40 - 50 years (42) followed by 30 - 40 years (12) and 20 - 30 years (4) respectively.

\begin{tabular}{|ccc|}
\hline $\begin{array}{c}\text { Variables } \\
\text { Subjective Global Assessment Scale }\end{array}$ & Survivors & $\begin{array}{c}\text { Non } \\
\text { Survivors }\end{array}$ \\
A & 25 & 2 \\
B & 21 & 3 \\
C & 2 & 7 \\
\hline Table 2. Outcome of Patient in ICU & \\
\hline
\end{tabular}

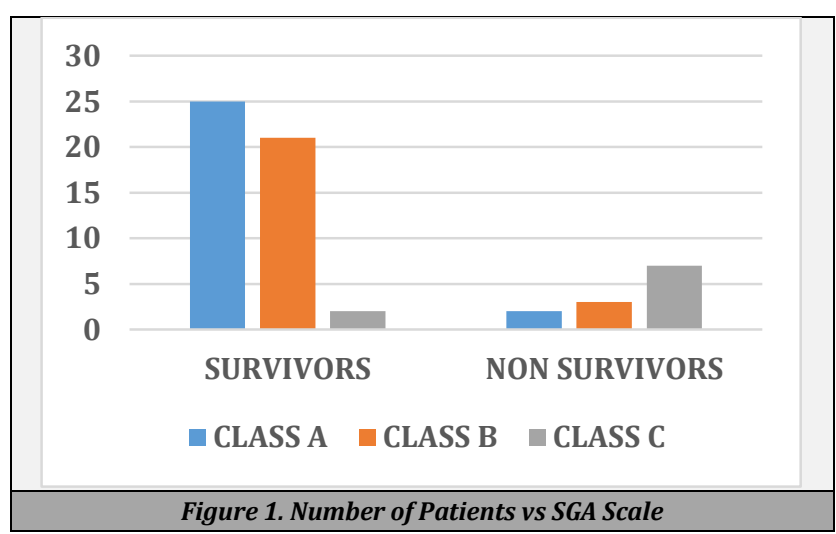

\begin{tabular}{|cccc|}
\hline Variables & \multicolumn{3}{c|}{ The Subjective Global } \\
& Assessment (SGA) Scale \\
Diagnosis & Class A & Class B & Class C \\
Stroke & $19(31.66 \%)$ & $3(5 \%)$ & - \\
Postoperative Respiratory Failure & $4(6.66 \%)$ & $4(6.66 \%)$ & $4(6.66 \%)$ \\
VAP ( Ventilator Associated Pneumonia) & $2(3.33 \%)$ & $8(13.33 \%)$ & $1(1.66 \%)$ \\
Sepsis & $2(3.33 \%)$ & $9(15 \%)$ & $4(6.66 \%)$ \\
\hline Table 3. Quality of Patients Who were Categorized under SGA Scale \\
\hline
\end{tabular}

Table no 3 shows the Quality of Patients which are categorized under SGA Scale. We classified the patients in class A, class B and class $\mathrm{C}$ as per the SGA score.

\section{DISCUSSION}

Statistics suggest that between $15 \%$ and $70 \%$ of hospital admitted patients are undernourished. Research have demonstrated a substantial prevalence of obesity in ICU patients ${ }^{13}$ Limited evidence from Indian hospitals show comparable low rates of feeding in the admitted patients, reflecting weak baseline nutritional status. ${ }^{16}$

SGA is a dietary evaluation tool that reflects on some aspects from medical background, such as recent weight change and food consumption, gastrointestinal issues with dietary consequences, cognitive capacity and stress strength, and subjective physical assessment of fat and muscle resources.

SGA is urged to consider patients who may benefit from dietary therapies. This is now deemed a gold standard technique for conducting such an analysis in the healthcare environment. ${ }^{17} \mathrm{SGA}$ is also becoming a practical approach to identify malnourished ICU patients with a greater probability of negative effects and a better predictive efficiency of ICU patients relative to other nutrients assessment tools. ${ }^{18}$ SGA 
may also complement essential nutritional tests in patients considered at risk of food insecurity. ${ }^{19}$

The clinicians were classified on the basis of SGA as well nourished (A), supposedly or moderately malnourished (B), or severely under nourished. We used SGA as a nutritional assessment method in this study because it was validated as reproducible and predictive of hospital - associated morbidity.

Out of 60 patients in our study, $45 \%$ were found to be well - nourished, $40 \%$ were found to be mild - moderately malnourished and $15 \%$ were found to be severely malnourished corresponding to SGA class A, B and C respectively. The overall mortality was $20 \%$ (12 out of 60 patients died). The incidence of mortality was higher in patients belonging to SGA class $\mathrm{C}$.

The results of the current research, becoming the observational form, may extend to other neurosurgical ICUs based in resource - constrained healthcare, particularly neighbouring countries in India and South Asia. ${ }^{20}$

\section{CONCLUSIONS}

Nutritional assistance is a critical factor that can negatively impact mortality and morbidity of intensive care patients. Malnutrition is normal in admitted neurosurgical ICU patients. Such patients often appear to be undernourished and have a negative impact on ICU results. Global evaluations are a valuable method for nutritional evaluation in neurosurgical patients in ICU and aid in the early diagnosis of malnutrition, which is linked with bad results in people with critical illness.

Financial or Other Competing Interests: None.

\section{REFERENCES}

[1] Lugli AK, Watteville AD, Hollinger A, et al. Medical nutrition therapy in critically ill patients treated on intensive and intermediate care units: a literature review. J Clin Med 2019;8(9):1395.

[2] Singh N, Gupta D, Aggarwal AN, et al. An assessment of nutritional support to critically ill patients and its correlation with outcomes in a respiratory intensive care unit. Respir Care 2009;54(12):1688-96.

[3] Reber E, Gomes F, Vasiloglou MF, et al. Nutritional risk screening and assessment. J Clin Med 2019;8(7):1065.

[4] Lim SL, Ong KC, Chan YH, et al. Malnutrition and its impact on cost of hospitalization, length of stay, readmission and 3 - year mortality. Clin Nutr 2012;31(3):345-50.

[5] Correia MITD, Campos ACL. Prevalence of hospital malnutrition in Latin America: the multicenter ELAN study. Nutrition 2003;19(10):823-5.
[6] Silva HG, Santos SO, Silva NO, et al. Nutritional assessment associated with length of inpatients' hospital stay. Nutr Hosp 2012;27(2):542-7.

[7] Norman K, Pichard C, Lochs H, et al. Prognostic impact of disease - related malnutrition. Clin Nutr 2008;27(1):5-15.

[8] Corkins MR, Guenter P, DiMaria-Ghalili RA, et al. Malnutrition diagnoses in hospitalized patients: United States, 2010. JPEN J Parenter Enteral Nutr 2014;38(2):186-95.

[9] Santarpia L, Contaldo F, Pasanisi F. Nutritional screening and early treatment of malnutrition in cancer patients. J Cachexia Sarcopenia Muscle 2011;2(1):27-35.

[10] Institute of Medicine (US). Committee on Nutrition Services for Medicare Beneficiaries. The role of nutrition in maintaining health in the nation's elderly: evaluating coverage of nutrition services for the medicare population. National Academy Press 2000.

[11] Carniel MP, Santetti D, Andrade JS, et al. Validation of a subjective global assessment questionnaire. J Pediatr (Rio J) 2015;91(6):596-602.

[12] Wakahara T, Shiraki M, Murase K, et al. Nutritional screening with subjective global assessment predicts hospital stay in patients with digestive diseases. Nutrition 2007;23(9):634-9.

[13] Detsky AS, McLaughlin JR, Baker JP, et al. What is subjective global assessment of nutritional status? JPEN J Parenter Enteral Nutr 1987;11(1):8-13.

[14] Singer P, Blaser AR, Berger MM, et al. ESPEN guideline on clinical nutrition in the intensive care unit. Clin Nutr 2019;38(1):48-79.

[15] Assessment (SGA) - CMTF - Canadian Malnutrition Task Force. https://nutritioncareincanada.ca/resources - and tools/hospital - care-inpac/assessment-sga

[16] Barbosa-Silva MCG, Barros AJD. Indications and limitations of the use of subjective global assessment in clinical practice: an update. Curr Opin Clin Nutr Metab Care 2006;9(3):263-9.

[17] Charlton K, Nichols C, Bowden S, et al. Poor nutritional status of older subacute patients predicts clinical outcomes and mortality at 18 months of follow-up. Eur J Clin Nutr 2012;66(11):1224-8.

[18] Aziz NASA, Teng NIMF, Hamid MRA, et al. Assessing the nutritional status of hospitalized elderly. Clin Interv Aging 2017;12:1615-25.

[19] Ahmed T, Hossain M, Mahfuz M, et al. Severe acute malnutrition in Asia. Food Nutr Bull 2014;35(Suppl 2):S14-26.

[20] Hejazi N, Mazloom Z, Zand F, et al. Nutritional assessment in critically ill patients. Iran J Med Sci 2016;41(3):171-9. 